\title{
Les élèves de lycée et la notion d'énergie
}

\section{B. bouldoires et R. Lefevre}

\section{(2) OpenEdition}

Journals

Édition électronique

URL : http://journals.openedition.org/trema/2329

DOI : 10.4000/trema.2329

ISSN : 2107-0997

\section{Éditeur}

Faculté d'Éducation de l'université de Montpellier

\section{Édition imprimée}

Date de publication : 1 mai 1993

Pagination : 49-67

ISSN : 1167-315X

\section{Référence électronique}

B. bouldoires et R. Lefevre, «Les élèves de lycée et la notion d'énergie », Tréma [En ligne], 3-4 | 1993, mis en ligne le 01 mai 1993, consulté le 19 avril 2019. URL : http://journals.openedition.org/ trema/2329 ; DOI : 10.4000/trema.2329

Ce document a été généré automatiquement le 19 avril 2019

Trema 


\title{
Les élèves de lycée et la notion d'énergie
}

\author{
B. bouldoires et $\mathrm{R}$. Lefevre
}

\section{Objectif général de la recherche ${ }^{1}$}

1 On se propose de préciser comment des élèves de la section F2 des Lycées (électronique) conçoivent et utilisent la notion d'énergie. Pour tenter de mettre en évidence certaines particularités de ces élèves, on compare leurs productions à celles d'autres élèves de l'enseignement général. On essaie également de caractériser l'évolution des "points de vue » en fonction du niveau (classes de Seconde, Première et Terminale).

\section{Problématique}

2 Ce compte-rendu de recherche comporte deux «volets» distincts bien que liés: l'un concerne l'exposé de résultats, l'autre est relatif à la méthodologie mise en œuvre.

3 En ce qui concerne les résultats, on se propose d'apporter des éléments de réponse à la question suivante : «A propos d'Energie, quelles sont les effets, sur des élèves de lycée, des deux variables : niveau scolaire et contenu d'enseignement?». La variable « niveau scolaire » possède ici trois modalités: Seconde, Première et Terminale. La variable «contenu d'enseignement» deux: «Enseignement de type physique appliquée ${ }^{2}$ et «Enseignement général de sciences physiques "³. Bien-sûr d'autres variables existent : les élèves ne sont pas répartis au hasard dans les différentes sections et leurs caractéristiques personnelles peuvent masquer les effets que l'on cherche à mettre en évidence.

4 En ce qui concerne la méthodologie, on se propose de répondre à la question suivante : «Le logiciel ALCESTE ${ }^{4}$ permet-il d'envisager l'analyse de questionnaires « ouverts », dans le cadre d'une recherche en didactique des sciences physiques?» Tenter de répondre à une telle question exige que l'on envisage d'abord une validation interne de l'outil (stabilité des résultats obtenus et possibilité de les interpréter) puis externe (non contradiction avec des résultats obtenus par une autre méthode, sur le même corpus). On 
se propose également de cerner les caractéristiques des questions qui, dans le domaine des recherches en didactique, sont « adaptées » à une analyse de type lexical.

\section{Méthodologie}

5 L'outil de recueil d'informations est un questionnaire comportant des questions «ouvertes». Son élaboration a été précédée d'entretiens qui ont permis de pointer quelques-unes des difficultés rencontrées par les élèves de 1ère F2. Il comprend cinq questions lexicales et une question graphique (non traitée ici) :

- Question $n^{\circ} 1$ : «L'énergie, qu'est-ce que cela évoque pour vous?»

- Question $n^{\circ} 2:$ «Une ampoule est reliée à une batterie par deux fils dont l'un comporte un interrupteur. Comment décririez-vous, en utilisant la notion d'énergie, ce qui se passe lorsqu'on ferme l'interrupteur?»

- Question $n^{\circ} 3$ : «On met des cubes de glace dans un bol d'eau chaude. Comment décririezvous, en utilisant la notion d'énergie, ce qui se passe alors?»

- Question $n^{\circ} 4:$ «Les freins d'une automobile garée en haut d'une rue en pente sont mal serrés. L'automobile dévale la rue et heurte un mur contre lequel elle s'écrase. Comment décririez-vous, en utilisant la notion d'énergie, ce qui se passe pendant cette scène ?»

- Question $n^{\circ} 5$ : «Essayez d'imaginer un slogan pour présenter l'énergie. »

- Question $n^{\circ} 6$ : «Essayez de faire ci-dessous un dessin qui illustre l'énergie. »

Idées générales ayant présidé à l'élaboration du questionnaire : on souhaite que les élèves expriment leurs conceptions telles qu'elles sont susceptibles d'apparaitre pendant la phase initiale d'une démarche de résolution de problème. L'électricité, la thermodynamique (en fait limitée à l'aspect calorimétrique) et la mécanique, sont abordées par trois questions.

Ordre des questions : deux questions non contraignantes $\left(n^{\circ} 1\right.$ et $\left.n^{\circ} 5\right)$ encadrent trois questions spécifiquement scientifiques. La cinquième question, destinée à recueillir l'idée maîtresse des élèves à propos d'énergie, sera-t-elle ou non influencée par le contexte «sciences physiques » des trois questions précédentes?

Enoncés des questions-problèmes: certaines précisions ne sont pas fournies car on souhaite vérifier l'hypothèse selon laquelle les élèves complètent un énoncé à partir de ce qu'ils connaissent de situations habituelles considérées comme pertinentes ${ }^{5}$. Les termes utilisés font partie du langage courant.

Conditions de recueil des informations : cette enquête s'est déroulée sur une période de trois semaines, à la fin de l'année scolaire 1990-91. Le questionnaire a été distribué aux élèves par le professeur de physique et rempli pendant l'heure de cours suivante. Les élèves, disposant de tout leur temps, ont mis entre 25 et 45 minutes pour répondre.

\section{Présentation de la population concernée par cette enquête}

\subsection{En quelques chiffres}

10 Deux lycées. Trois niveaux (2ème, 1ère et Terminale). Six sections (TSA, F2, F'2, E, S, C, et Pro.). Treize classes (plus deux groupes d'élèves de 2ème issus de différentes classes). 
Nombre d'élèves dont les réponses constituent le corpus d'analyse : 318 . Sur les 331 questionnaires remplis, 13 sont hors sujet ou inexploitables.

\subsection{Organisation résultant du choix des variables}

11 Le corpus constitué par l'ensemble des réponses a été découpé en six groupes aux fins d'analyse. Le découpage par niveau et par section est représenté sur la figure ci dessous.

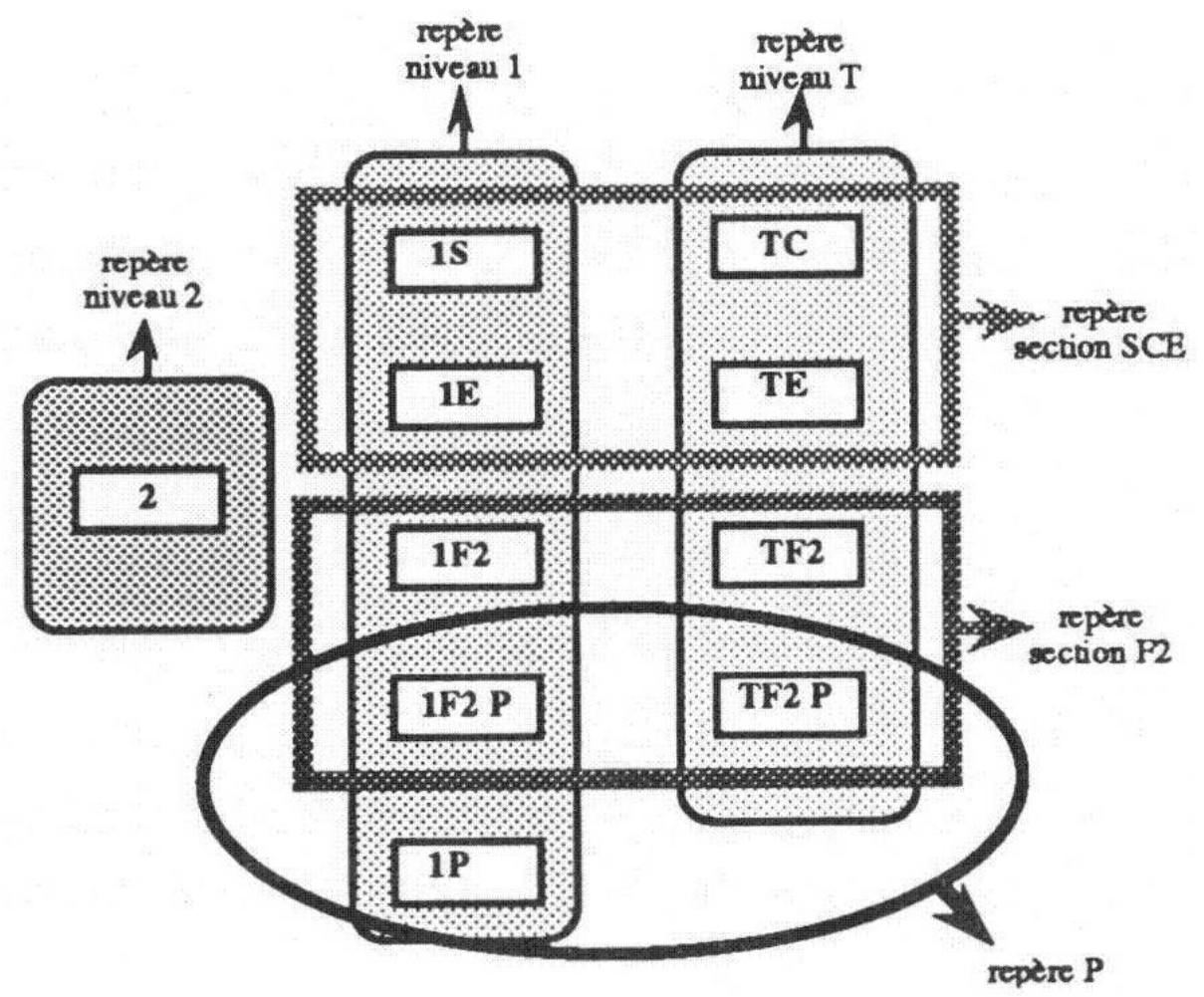

Les effectifs des six groupes ainsi formés sont les suivants :

- repère section $\mathrm{F} 2: 113$

- repère («section ») $\mathrm{P}: 48$

- repère niveau $1: 118$

- repère section SCE : 96

- repère niveau $2: 97$

- repère niveau $\mathrm{T}: 103$

\section{Mise en forme du corpus}

\subsection{Choix effectués pour la transcription du corpus}

13 Même lorsque les réponses sont écrites, leur transcription s'accompagne de choix. Les élèves utilisent parfois des abréviations ( $E$ ou nrj pour énergie, e- pour électron par exemple) ou des symboles mathématiques $(=>;+;=$...) à la place de certains mots ou de certaines expressions. Nous avons donc remplacé ces signes par les mots et expressions correspondants. Il y a là œuvre de traduction. Par exemple « => a été traduit par « implique », « ce qui implique », « entraîne ", « a pour conséquence » [...] suivant ce qui paraissait être le plus approprié dans le contexte, bien qu'on ne puisse jamais être tout à 
fait certain de la signification que l'élève lui attribuait. Nous avons reconstitué la ponctuation absente ou fantaisiste dans la mesure où cela nous paraissait nécessaire et

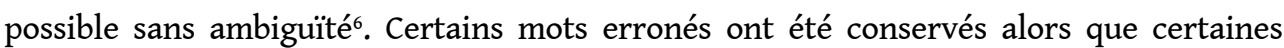
orthographes fantaisistes ont été rectifiées. Par exemple, « cinématique » utilisé à la place de " cinétique » a été conservé, alors que " cynétique » a été remplacé par « cinétique ». Des phrases incompréhensibles ont été relevées en l'état. Des formules comme $\mathrm{E}=1 /{ }_{2} \mathrm{mV}^{2}$, ont été remplacées par : $\mathrm{E}$ égale un demi de $\mathrm{m}$ que multiplie $\mathrm{V}$ au carré. Ces adaptations permettent au logiciel de prendre en compte des informations qui, sinon, auraient été ignorées.

\subsection{Codage des réponses suivant les normes d'ALCESTE}

Chaque réponse est précédée d'un certain nombre de codes correspondant à des variables d'identification de la question et du répondant :

$\mathrm{N}^{\circ}$ question : ${ }^{*} \mathrm{q}-\mathrm{a}[\mathrm{a}=1,2,3,4$ ou 5$]$

Niveau : ${ }^{*}$ ni_b $[b=2$ pour Seconde, 1 pour Première et $T$ pour Terminale $]$

Section : *sec_c [c = T pour TSA, F2 , E, C et P pour Professionnel]

Particularité : *pa_d [d = N pour normal, P pour Prime ou professionnel]

Age : *ag_e [e =15, 16, 17, 18, 19, 20, 21 ; âge en années entières révolues]

Lycée : ${ }^{*} \mathrm{~L}_{-} \mathrm{f}[\mathrm{f}=\mathrm{A}$ ou $\mathrm{B}]$

On a choisi de séparer un certain nombre de variables pour effectuer ensuite certains tris. Ainsi *pa_P se rapporte à tous les élèves qui sont passés par un lycée professionnel, c'està-dire les élèves de F2 « prime » et ceux de Bac Pro.

\section{Premier résultat}

Très peu d'élèves ont noté que les renseignements fournis dans les énoncés des questions ne permettaient pas de savoir avec exactitude ce qui allait se passer.

Par exemple, en ce qui concerne la deuxième question :

$0051{ }^{*}$ q_2 2 *ni_T *sec_F2 *pa_N *ag_18 *L_A : «En général, si l'ampoule est adaptée à la batterie, elle s'allume.... »

0219 *q_2 *ni_1 *sec_F2 *pa_N *ag_18 *L_B : « Lorsqu'on ferme l'interrupteur, l'énergie se déplace dans les fils et permet donc à l'ampoule de s'allumer mais lorsque la batterie sera déchargée, l'ampoule n'éclairera plus, il faudra donc recharger la batterie ... »

0279 *q_2 *ni_2 *sec_N *pa_N *ag_17 *L_A : «L'énergie passe dans le circuit pour alimenter les ampoules. II faut que la batterie soit chargée. »

0281 *q_2 *ni_2 *sec_N *pa_N *ag_15 *L_A : «L'énergie circule dans les fils lorsque l'interrupteur est fermé et lorsque la batterie est chargée. L'ampoule éclaire. »

$0286{ }^{*}$ q_2 ${ }^{*}$ ni_2 * sec_N * ${ }^{*}$ pa_N *ag_15 * L_A : « Normalement l'ampoule se met à briller mais il faut que la batterie soit chargée... »

Moins de $2 \%$ des élèves qui ont effectivement répondu à cette question expriment ainsi une hypothèse sur le fonctionnement de la batterie. Il y a donc très peu de raisonnements déductifs sur hypothèses qui marqueraient un peu de « recul spontané $»^{7}$ par rapport au problème. On peut tenter d'interpréter cela en supposant que, dans leur grande majorité, 
les élèves complètent les énoncés des questions-problèmes par des informations, issues de leurs expériences quotidiennes ou scolaires, auxquelles ils accorderaient un statut de certitude et non pas d'hypothèse. Le degré de pertinence ${ }^{8} \mathrm{~d}^{\prime}$ un dysfonctionnement de la batterie étant peut-être fonction des expériences réellement vécues par les uns et les autres ${ }^{9}$.

\section{Analyse du contenu des réponses des élèves en utilisant ALCESTE}

\subsection{Limites du problème que l'on se propose d'aborder}

19 Il ne s'agit pas de donner des résultats définitifs concernant les caractéristiques des réponses et encore moins, bien sûr, des élèves. Les limites d'une approche purement lexicale sont mentionnées par L. Lebart et A. Salem (1988, p. 17). On se propose simplement de rechercher quelle « image » peut être obtenue à l'aide d'un tel logiciel. Il conviendra de discuter la validité de cette image en réfléchissant à sa cohérence interne ainsi qu'aux diverses instabilités susceptibles d'apparaître.

\subsection{Résultats par question}

\section{Question $n^{\circ} 1$}

«L'énergie, qu'est-ce que cela évoque pour vous?»

- En ce qui concerne le corpus complet, constitué de 310 réponses, plusieurs plans d'analyse ont été utilisés. On a choisi d'en retenir deux, représentatifs des deux analyses « extrêmes » qu'il est possible d'entreprendre à l'aide du logiciel ALCESTE : un plan NI, caractérisé par une simple classification des réponses (non découpées en unités de contexte élémentaires si leur longueur est inférieure à dix lignes, ce qui est le cas ici), en tenant compte des formes d'origine, un plan SX, caractérisé par une double classification des u.c.e. et conservation des seules classes «stables", en tenant compte des formes réduites ${ }^{10}$.

21 - Résultats de l'analyse obtenue à l'aide d'un plan NI/12 : le dendogramme de l'analyse descendante hiérarchique et les profils des différentes classes d'analyse permettent de faire apparaître trois "idées" principales, caractéristiques (au sens d'ALCESTE) des réponses fournies par les élèves à cette question. Ces « idées » principales regroupent des « idées secondaires ». Le tout peut être résumé de la manière suivante :

1 Pas de vie pour l'homme sans énergie.

1.1 Importance des sources naturelles d'énergie (en particulier pour l'économie).

1.2 L'énergie est indispensable : pas de vie sans énergie (au sens large de la nature).

1.3 L'énergie est utile à la vie des hommes (progrès, confort).

2 L'énergie c'est ce qui permet de faire fonctionner, de fournir du travail.

2.1 L'énergie fait fonctionner des objets, des systèmes, des appareils.

2.2 L'énergie présente un caractère de généralité.

3 Les différentes énergies. 
3.1 Les formes d'énergie (cinétique, potentielle ...).

3.2 Différentes sortes d'énergie (solaire, éolienne, hydaulique ...)

3.3 La chaleur.

22

23

A ces trois idées, il convient d'ajouter la «classe » des formules. On obtient ainsi un premier tableau d'ensemble des différents types de réponses.

- Résultats de l'analyse obtenue à l'aide d'un plan SX14-12/12: les profils des quatre classes d'analyse obtenues et les u.c.e. les plus caractéristiques suggèrent l'interprétation suivante :

$7^{\text {ère }}$ classe (110 u.c.e.) : Liste des différentes formes d'énergie.

2ème classe (114 u.c.e.) : L'énergie comme besoin, quelque chose dont on ne peut pas se passer.

$3^{\text {ème }}$ classe (72 u.c.e.) : LES SOURCES d'ÉNERgIE.

$4^{\text {ème }}$ classe (20 u.c.e.) : $\mathbf{E}=\mathbf{M} \mathbf{~ c}^{2}$.

Les deux "images ", obtenues à l'aide de deux plans d'analyse très différents, étant comparables, il semble que l'on soit en droit de valider ces résultats. On peut compléter cette approche à l'aide d'une analyse factorielle des correspondances. Trois axes factoriels permettent « d'expliquer » $100 \%$ de l'inertie. Le premier axe factoriel (48\% de l'inertie) semble opposer les formules à (presque) tout le reste. Le deuxième axe factoriel (30\% de l'inertie) semble opposer l'utilisation, associée à l'Homme et à la vie, aux formes d'énergie. Le troisième axe factoriel ( $22 \%$ de l'inertie) semble opposer sources naturelles d'énergie à utilisation de l'énergie, en passant par les différentes formes d'énergie.

Les niveaux et les sections sont faiblement corrélés aux quatre classes d'analyse. Les approches de la notion d'énergie seraient donc relativement indépendantes des caractéristiques scolaires des élèves de lycée.

- Pour tenter cependant de déceler des spécificités qui n'apparaissent pas dans l'analyse du corpus complet ${ }^{11}$, on a traité séparément les sous-corpus constitués des réponses de tous les élèves d'une section ou d'un niveau. Même si ces réponses constituent un continuum au sein duquel tout découpage est en partie arbitraire, il semble intéressant de disposer de points de repères voire même simplement de quelques «points d'interrogations». Ce sont ceux-ci que l'on se propose de faire émerger. Les interprétations suggérées par les résultats obtenus ${ }^{12}$ sont les suivantes : 


\begin{tabular}{|l|l|l|}
\cline { 2 - 3 } \multicolumn{1}{c|}{} & \multicolumn{1}{c|}{ Caractéristiques principales } & Caractéristiques secondaires \\
\hline sec_F2 & $\begin{array}{l}\text { L'énergie est présentée par } \\
\text { une liste de ses différentes } \\
\text { formes. }\end{array}$ & $\begin{array}{l}\text { L'énergie comme "force" } \\
\text { capable de faire fonctionner } \\
\text { des appareils, elle est souvent } \\
\text { associée au mouvement, elle } \\
\text { est nécessaire à la vie et } \\
\text { associée au progrès et au } \\
\text { confort. }\end{array}$ \\
\hline sec_SCE & $\begin{array}{l}\text { L'énergie c'est ce qui permet } \\
\text { de faire un travail. }\end{array}$ & Les formes d'énergie. \\
\hline
\end{tabular}

\begin{tabular}{|c|l|l|}
\hline "sec_P" & $\begin{array}{l}\text { Les différents "types" } \\
\text { d'énergie. }\end{array}$ & $\begin{array}{l}\text { Les sources d'énergie-la } \\
\text { nature, la vie. }\end{array}$ \\
\hline ni_2 & $\begin{array}{l}\text { L'énergie comme force qui } \\
\text { permet de "faire des choses". }\end{array}$ & $\begin{array}{l}\text { Plusieurs sortes d'énergie, } \\
\text { avec un flou important dans la } \\
\text { catégorisation (énergie pneu- } \\
\text { matique), confusion énergie/ } \\
\text { matière (l'énergie c'est le } \\
\text { glucose), confusion énergie/ } \\
\text { source d'énergie. }\end{array}$ \\
\hline ni_1 & $\begin{array}{l}\text { L'énergie est nécessaire à la } \\
\text { vie, au confort, au fonctionne- } \\
\text { ment de différents systèmes } \\
\text { techniques. }\end{array}$ & $\begin{array}{l}\text { Différents types, sortes ou } \\
\text { formes d'énergie(s). }\end{array}$ \\
\hline ni_T & $\begin{array}{l}\text { Différents aspects ou formes } \\
\text { de l'énergie avec prise en } \\
\text { compte de transformations. }\end{array}$ & $\begin{array}{l}\text { Besoin, moyen indispensable, } \\
\text { l'énergie don de la nature } \\
\text { contribue à la vie. }\end{array}$ \\
\hline
\end{tabular}

En ce qui concerne les sections, on peut remarquer une différence assez nette entre F2 et SCE. Les élèves de la section F2 semblent principalement aborder l'énergie par l'aspect diversité (les différentes formes d'énergie), les élèves des sections $\mathrm{S}, \mathrm{C}$ et $\mathrm{E}$ semblent au contraire avoir une approche plus unitaire en donnant prioritairement une définition générale. En ce qui concerne les niveaux, on peut remarquer qu'en Terminale les élèves semblent privilégier les différentes formes d'énergie, l'association énergie-vie étant présente au second plan seulement. En 1ère, les élèves semblent évoquer d'abord l'utilité de l'énergie, en liaison avec la vie et l'Homme, le point de vue classificatoire ne venant qu'en deuxième lieu. En Seconde, ces deux approches sont affectées de deux "poids " comparables et la catégorisation est particulièrement floue (les aspects économiques, techniques et scientifiques ne sont pas séparés). On peut également noter une certaine 
subtilité de l'approche des élèves de Terminale: les différentes formes de l'énergie semblent bien être perçues comme différents aspects, ce qui sous-entend la reconnaissance d'une certaine unité derrière la diversité. Ce point de vue semble associé à la prise en compte des transformations.

\section{Question $n^{\circ} 2$}

« Une ampoule est reliée à une batterie par deux fils dont l'un comporte un interrupteur. Comment décririez-vous, en utilisant la notion d'énergie, ce qui se passe lorsqu'on ferme l'interrupteur?»

Les analyses du corpus de cette question, constitué de 310 réponses, révèlent une forte instabilité des résultats en fonction des plans d'analyse choisis, de leurs paramètres ainsi que de la simple mise en forme du texte.

- A titre de première approche, on peut cependant résumer dans un tableau l'ensemble des résultats obtenus ${ }^{13}$ pour le corpus complet, à l'aide d'un plan NI/12 suivant une procédure identique à celle utilisée pour les réponses à la première question :

\begin{tabular}{l|c|c} 
"idées" & u.c.e. en \% & "idée" associée à \\
\hline Le courant fait briller la lampe & 47 & ni_2 \\
Transformation d'énergie & 37 & ni_T et sec_F2 \\
Approche diversifiée & 14 & Sec_SCE
\end{tabular}

- On a également traité séparément les sous-corpus constitués des réponses des élèves de chaque section et de chaque niveau. Les principaux résultats obtenus ${ }^{14}$ sont rassemblées dans le tableau suivant :

\begin{tabular}{|c|c|c|}
\hline & Caractéristiques principales & Caractéristiques secondaires \\
\hline sec_F2 & $\begin{array}{l}\text { "L'ampoule est alimentée en } \\
\text { énergie électrique (consom- } \\
\text { mation) et elle produit } \\
\text { (l'ampoule) une énergie } \\
\text { lumineuse et calorifique, il y } \\
\text { a transformation d'énergie" ; } \\
84 \text { u.c.e., soit } 61 \% \text { des u.c.e. } \\
\text { classées. }\end{array}$ & $\begin{array}{l}\text { "Le circuit est fermé lorsqu'on } \\
\text { ferme l'interrupteur. Le } \\
\text { courant passe et l'ampoule } \\
\text { s'allume" } \\
53 \text { u.c.e., soit } 39 \% \text { des u.c.e. } \\
\text { classées. }\end{array}$ \\
\hline Sec_SCE & \multicolumn{2}{|c|}{$\begin{array}{l}\text { "Dans l'ampoule, le filament ayant une grande résistance, il y } \\
\text { a échauffement de ce filament (énergie thermique), cet } \\
\text { échauffement porte le filament à incandescence " ; } 42 \text { u.c.e., } \\
\text { soit } 30 \% \text { des u.c.e. classées. Corrélé au niveau 1ère. } \\
\text { "... La chaleur se disperse à tout jamais dans la pièce sous } \\
\text { forme d'augmentation de désordre des particules " et "L'éner- } \\
\text { gie circule sous forme d'électricité mais est libérée sous forme } \\
\text { de chaleur au niveau de l'ampoule "; } 33 \text { u.c.e., soit } 24 \% \text { des } \\
\text { u.c.e. classées. Corrélé au niveau Terminale. } \\
\text { "... Lorsqu'on ferme l'interrupteur, il passe une énergie } \\
\text { électrique de la batterie vers l'ampoule qui peut s'allumer "; } \\
65 \text { u.c.e., soit } 46 \% \text { des u.c.e. classées. }\end{array}$} \\
\hline
\end{tabular}




\begin{tabular}{|c|c|c|}
\hline$" \sec { }^{p} p$ & \multicolumn{2}{|c|}{ 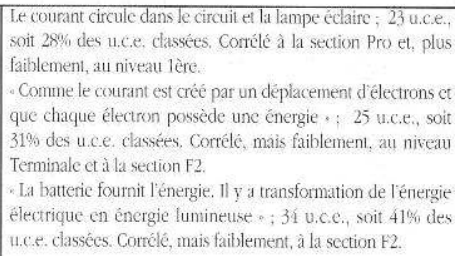 } \\
\hline ni__2 & $\begin{array}{l}\text { Lampoule sallume: } \\
96 \text { u.ce., soit } 73 \% \text { des u.c.e. } \\
\text { classees. }\end{array}$ & $\begin{array}{l}\text { lles électrons provoquent la } \\
\text { luminosite de la lampe en } \\
\text { traversant le filament puis } \\
\text { retournent à la pile par la } \\
\text { borne plus } \\
35 \text { u.c.e., soit } 27 \% \text { des u.c.e. } \\
\text { classées. }\end{array}$ \\
\hline ni_1 & $\begin{array}{l}\text { La batterie délivre l'énergie } \\
\text { qui permet à lampoule de } \\
\text { sallumer: } 118 \text { u.ce, soit } 69 \% \\
\text { des u.c.é, classées. } \\
\text { L'énergie électrique se } \\
\text { transforme en énergie lu- } \\
\text { minetuse; } \\
155 \text { u.c.e., soit } 80 \% \text { des u.ce. } \\
\text { classécs. }\end{array}$ & 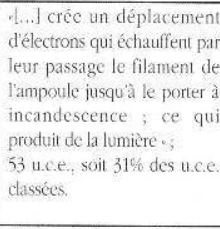 \\
\hline ni_T & & 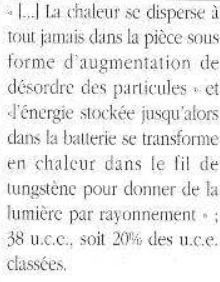 \\
\hline
\end{tabular}

A la lecture de ce tableau, les résultats les plus notables nous paraissent être :

- la diversité des approches des élèves de la section SCE,

- la progression nette du point de vue énergétique de la Seconde à la Terminale.

Une analyse sémantique des réponses à cette question a commencé. Les premiers résultats suggèrent que le problème essentiel des élèves pourrait résulter de l'intrication de deux modèles, un modèle descriptif de type "circuit électrique $»^{15}$ et un modèle descriptif de type "chaîne énergétique $~^{16}$. La gestion de cette double modélisation n'étant pas toujours maîtrisée. On peut également remarquer que cette question, probablement la plus « inadaptée » à un traitement par le logiciel ALCESTE, est justement celle qui est relative à une partie des sciences physiques pour laquelle tous les élèves possèdent à peu près les mêmes bases lexicales.

\section{Question $n^{\circ} 3$}

"On met des cubes de glace dans un bol d'eau chaude. Comment décririez-vous, en utilisant la notion d'énergie, ce qui se passe alors?»

- Une analyse à l'aide d'un plan «standard » SX14-16/10 permet d'obtenir un découpage du corpus complet, constitué de 297 réponses, en quatre classes que l'on peut caractériser par les phrases suivantes :

$7^{\text {ère }}$ classe (fortement corrélée au niveau Seconde - 155 u.c.e., soit $47 \%$ ) : L'EAU CHAUDE FAIT FONDRE LA gLACE ET ELLE SE REFROIDIT.

$2^{\text {ème }}$ classe (corrélée au niveau Terminale - 63 u.c.e., soit 19\%) : Changement d'état à zéro degré Celsius : le solide se transforme en liquide. 
$3^{\text {ème }}$ classe (fortement corrélée au niveau Première et un peu moins à la section SCE - 71 u.c.e., soit : $22 \%$ ) : Le MILIEU LE PLUS ChAUd CÈde de l'ÉNERgIE JUSQU'À Ce QU'IL Y AIT ÉQUILIBRE THERMIQUE.

$4^{\text {ème }}$ classe (corrélée, mais faiblement au niveau Terminale - 38 u.c.e., soit $12 \%$ ) : L'énergie est perdue, libérée, se transmet [...] sous forme de chaleur.

La quatrième classe est la seule qui ne soit pas très stable lorsque l'on fait varier les paramètres du plan d'analyse.

Il semble que cette question permette de différencier les élèves en fonction de leur niveau scolaire $2^{\text {ème }}, 1^{\text {ère }}$ et Terminale. Pour les sections, il est remarquable que les élèves de F2 ne soient associés à aucune classe d'analyse. Cela incite à mener une étude spécifique du sous-groupe F2.

\begin{tabular}{|c|c|c|}
\hline & Caractéristiques principales & Caractéristiques secondaires \\
\hline sec_f2 & $\begin{array}{l}\text { Leau chaude (ou son énergic } \\
\text { calorifique) fait fondre les } \\
\text { cubes de glace. }\end{array}$ & $\begin{array}{l}\text { La différence de température } \\
\text { provoque une réaction entre } \\
\text { les énergies des deux milicux } \\
\text { jusqu'à équilibre. }\end{array}$ \\
\hline sec_SCE & $\begin{array}{l}\text { - L'énergie calorifique fournie } \\
\text { aux glaconss les fait fondre et } \\
\text { l'eau se refroidit. } \\
\text { - Il y a transfert du milieu } \\
\text { chaud vers le milieu froid (ou } \\
\text { échange entre les deux } \\
\text { milieux). }\end{array}$ & $\begin{array}{l}\text { La chaleur perdue par leau } \\
\text { chaude et recue par les cubes } \\
\text { de glace provoque la fonte de } \\
\text { ces derniers (les chaleurs } \\
\text { perdue et reçue étant égales). } \\
\text { Les molécules transmettent } \\
\text { leur énergie cinétique d'agi- } \\
\text { tation. Le mélange de deux } \\
\text { corps à températures dif- } \\
\text { férentes se traduit, à léqui- } \\
\text { libre, par un thangement de } \\
\text { létat solide à létat liquuide. }\end{array}$ \\
\hline "sec_p" & $\begin{array}{l}\text { Les cubes de glace fondent } \\
\text { dans le bol deau chaude. }\end{array}$ & $\begin{array}{l}\text { II y a deux énergies qui } \\
\text { saffrontent, s'annulent I... } \\
\text { jusquáa léquilibre. }\end{array}$ \\
\hline ni_2 & $\begin{array}{l}\text { L'cau chaude fait fondre la } \\
\text { glace. }\end{array}$ & $\begin{array}{l}\text { Il y a fonte de la glace et leau } \\
\text { devient plus froide. }\end{array}$ \\
\hline ni_1 & $\begin{array}{l}\text { Lénergie calorifique de l'eau } \\
\text { fait fondre les cubes de glace } \\
\text { (ou les glaçons). }\end{array}$ & $\begin{array}{l}\text { Il y a un transfert dénergie } \\
\text { thermique entre les deux } \\
\text { corps. La glace recoit de } \\
\text { l'énergie sous forme de } \\
\text { chaleur. }\end{array}$ \\
\hline ni_t & $\begin{array}{l}\text { L'énergie calorifique (ou la } \\
\text { chaleur) de l'eau chaude va } \\
\text { faire fondre les cubes de } \\
\text { glace. }\end{array}$ & $\begin{array}{l}\text { Les molécules du liquide (ou } \\
\text { particules ou atomes) trans- } \\
\text { mettent leur énergie cinétique } \\
\text { aux molécules de la glace. }\end{array}$ \\
\hline
\end{tabular}

- Les corpus constitués des réponses des élèves de chaque sous-groupe «section » ou "niveau" ont été analysés séparément. Les interprétations suggérées par les résultats obtenus sont rassemblées dans le tableau précédent.

On pourrait synthétiser ces résultats de la manière suivante : les réponses des élèves des sections $\mathrm{F} 2$ et $\mathrm{P}$ semblent très proches de celles des élèves du niveau Seconde; ces réponses expriment souvent un simple constat. Cependant la notion d'équilibre n'apparaît pas au niveau Seconde alors qu'on la rencontre en F2. Les élèves de la section SCE offrent des réponses diversifiées qui semblent refléter les contenus des programmes : transfert, équilibre, changement d'état ect... avec une quantification des échanges absente ailleurs. Les élèves des niveaux Première et Terminale semblent donner des réponses voisines, avec cependant une prise en compte plus marquée de l'aspect « microscopique » en Terminale. En fait, dans ce cas, une étude par sous-groupe n'apporte pas grand-chose de plus qu'une analyse du corpus complet. 


\section{Question $n^{\circ} 4$}

40 "Les freins d'une automobile garée en haut d'une rue en pente sont mal serrés. L'automobile dévale la rue et heurte un mur contre lequel elle s'écrase. Comment décririez-vous, en utilisant la notion d'énergie, ce qui se passe pendant cette scène ?» Le corpus d'analyse de cette question comporte 264 réponses. Un plan SX212-16/12 a permis d'obtenir les résultats suivants :

$7^{\text {ère }}$ classe (très fortement corrélée au niveau seconde - 162 u.c.e., soit : 44 \%) : LE POIDS Comme CaUse.

$2^{2 \text { ème }}$ classe (très fortement corrélée à la section SCE - 105 u.c.e., soit $28 \%$ ) : Transformation de l'énergie potentielle en énergie cinétique.

$3^{\text {ème }}$ classe (fortement corrélée à la section F2 - 103 u.c.e., soit: $28 \%$ ): DESCRIPTION « Centrée » SUR L'augmentation de LA Vitesse.

42 Dans ce cas, les classes sont fortement corrélées aux groupes d'élèves. Il semble bien que chaque groupe puisse être caractérisé par ce que M. Reinert (1990) appelle « un univers de pensée " ou un "monde». Cependant les profils des classes sont assez difficiles à résumer, sauf en ce qui concerne la classe $n^{\circ} 2$. Pour les élèves de SCE l'univers de pensée utilisé est celui de la conservation de l'énergie mécanique ou de la transformation d'énergie potentielle en énergie cinétique. On constate bien là l'influence du programme de sciences physiques d'autant plus que la situation est très proche de celles abordées par les problèmes "standards "! En ce qui concerne les élèves de Seconde, on retiendra l'influence du poids et des freins mal serrés. Il semble que ces élèves essaient d'appliquer également ce qu'ils ont étudié pendant l'année scolaire en physique : la notion de force (forces de frottement, pesanteur, équilibre etc...). Les élèves de F2, quant à eux, semblent surtout caractérisés par une description issue de la vie courante : la voiture prend de la vitesse. Ces élèves n'essaient pas d'expliquer ce qui se passe en utilisant leurs connaissances scientifique $»^{17}$. En ce qui concerne les classes d'analyse $N^{\circ} 1$ et 3 , certaines u.c.e. caractéristiques proviennent d'élèves qui appartiennent à des groupes non fortement corrélés à ces mêmes classes. On peut également remarquer que des réponses issues des élèves de SCE se retrouvent dans toutes les classes car ces élèves procèdent souvent en plusieurs étapes successives : une étape descriptive en termes de forces et de rupture d'équilibre ${ }^{18}$, suivie d'une étape descriptive en terme d'augmentation de la vitesse de l'automobile ${ }^{19}$ qui débouche sur la prise en compte d'une transformation d'énergie potentielle en énergie cinétique ${ }^{20}$.

En ce qui concerne les élèves de Seconde, leurs réponses présentent deux «centres d'intérêt principaux»: «les forces (et en particulier le poids de la voiture) » et «les freins ", ces deux " centres d'intérêt » étant bien sûr largement complémentaires. En ce qui concerne les élèves de F2, leurs réponses présentent également deux «centres d'intérêt principaux»: «la vitesse de la voiture » et "le mur contre lequel celle-ci s'écrase ». Il convient cependant de remarquer que, suivant les paramètres choisis pour le plan SX, ces « centres d'intérêt » émergent plus ou moins nettement de l'analyse.

Les indications fournies par une analyse du corpus complet sont suffisamment nettes ici pour qu'il ne soit pas nécessaire d'entreprendre une étude par sous-groupe.

On peut conclure ce paragraphe par deux remarques :

- aucun élève n'a choisi un référentiel pour répondre à cette question, 
- aucun élève n'a envisagé la conservation globale de l'énergie (même en se limitant à la somme : énergie mécanique plus énergie calorifique) complètement opérationnel.

\section{Question $n^{\circ} 5$} $\mathrm{n}^{\circ} 5$, il ne semble pas y avoir de différences très significatives entre les élèves. Cependant, en ce qui concerne la question $n^{\circ} l$, les points de vues «évités » semblent être plus caractéristiques que les points de vue "adoptés ", ce qui impose de descendre, pour les analyses, au niveau des sous-groupes. En ce qui concerne les questions $n^{\circ} 3$ et $n^{\circ} 4$, les différences entre les élèves se font sentir et il semble bien qu'elles soient liées aux différences qui existent dans les programmes, soit entre les différentes sections (SCE et F2), soit entre les différents niveaux (2, 1 et $\mathrm{T})$.

- Qu'apporte ALCESTE pour le traitement des réponses à une question «inadaptée ", comme la question $n^{\circ} 2$ ? Les différences lexicales relevées par les analyses font apparaître une certaine image. Même si ses contours restent flous, cela constitue une première approche suggérant des pistes qui peuvent être explorées par d'autres méthodes.

53 - Que faire pour aller plus loin, c'est à dire obtenir une autre « image » des réponses aux questions « inadaptées »? Il semble bien qu'il faille changer d'approche et substituer une approche sémantique à cette approche lexicale. Cette approche sémantique est souvent considérée comme réductrice : le post-codage ne permettant pas de restituer toutes les subtilités des réponses (Lebart L. et Salem A., 1988, en particulier page 15). On peut également se demander si le flou associé à la série d'analyses effectuées sur le corpus des réponses à la deuxième question ne traduit pas une réalité qui serait que les élèves répondent justement de manière floue ${ }^{22}$. Ce flou pourrait résulter, comme le suggèrent les premiers résultats d'une approche sémantique, d'une superposition de deux modèles explicatifs ( « circuit électrique » et « chaîne énergétique »). Le fait que l'articulation entre 
ces deux modèles ne soit pas maîtrisée, pourrait « expliquer » la sensibilité des réponses des élèves à des questions identiques quant au fond mais présentées de manières différentes quant à leur forme. De petites variations pourraient modifier les degrés de pertinence associés à chaque modèle, orientant ainsi l'approche du problème dans l'une ou l'autre voie.

- Tentative de caractérisation d'une utilisation d'ALCESTE dans le cadre de recherches en didactique des sciences physiques.

- Univers de pensée et conceptions: Comment peut-on apprécier les possibilités d'utilisation du logiciel ALCESTE dans le cadre de telles recherches? Revenons un peu sur l'hypothèse initiale qui sous-tend la création de ce logiciel. Comme le rappelle son concepteur, cette hypothèse consiste à considérer que le contenu lexical du discours est caractéristique de l'univers de pensée du locuteur. Cela semble pouvoir être admis sans problème lorsqu'on souhaite caractériser la réponse à une question ouverte "générale " portant sur des opinions. Dans le cas de cette enquête, les questions $\mathrm{n}^{\circ} 1$ et $\mathrm{n}^{\circ} 5$ peuvent être considérées comme relevant de cette catégorie. Des élèves qui répondent à la première question en donnant une liste de formes d'énergie et ceux qui citent des formules, ou encore ceux qui font référence à la vie, ne se placent pas dans la même perspective. C'est également vrai pour la cinquième question, le slogan. La liberté laissée à l'élève étant importante, ses choix lexicaux semblent pouvoir être considérés comme caractéristiques de son approche. Il en va tout autrement en ce qui concerne les questions $\mathrm{n}^{\circ} 2,3$ et 4 . Là, le problème posé par la question fixe un cadre très contraignant. Les termes utilisés par les uns et les autres, dans leurs réponses, sont en partie bornés par l'enseignement reçu. A la limite, il est possible de construire deux réponses qui, en utilisant les mêmes termes, ont deux significations différentes ${ }^{23}$. Il semble bien qu'il y ait là un problème de fond lié à l'approche lexicale dans le cadre d'une recherche en didactique : dès que l'on s'intéresse aux conceptions relatives à un domaine disciplinaire précis, le vocabulaire se standardise. Cela pourrait expliquer pourquoi on a été obligé d'introduire plus tôt que prévu une étape interprétative ${ }^{24}$. L'outil ALCESTE ne semble pas devoir être remis en cause en tant que tel, mais il est possible que l'on soit en fait confronté à une difficulté conceptuelle. Celle-ci pourrait résulter d'une certaine confusion entre « univers de pensée » (peut-être entendu comme une représentation au sens des psychologues) et «conceptions» (du domaine des didactiques). Dans certains cas, lorsque le discours est produit en réponse à une question générale, la distance conceptuelle entre "univers de pensée » et "conception » est faible car le locuteur s'engage « librement » dans son propre univers de pensée. Mais, au contraire, lorsque le discours porte sur un domaine précis, cette distance peut devenir importante et il devient alors illusoire de chercher à obtenir des classes lexicales très fortement corrélées à des « conceptions $»^{25}$.

- Etudes des limites de validité des images obtenues à l'aide d'ALCESTE :

57 Tous les plans ne convenant pas pour étudier un corpus donné, il semble bien qu'il ne faille pas se contenter d'un seul plan d'analyse au cours d'une recherche.

On a essayé de savoir combien de réponses à une question devaient être rassemblées pour que les résultats puissent être considérés comme fiables. Dans ce but, des analyses ont été effectuées, à l'aide d'un même plan NI/12, sur le corpus de la deuxième question ${ }^{26}$. Et cela, dans trois cas : a) Corpus " complet », b) Corpus constitué des seules réponses " paires ", c) Corpus constitué des seules réponses « impaires ». Au delà de quelques différences, les interprétations convergent. Cependant une classe « disparaît » des résultats d'analyse des 
《demi-corpus $~_{27}$. Il faut donc, pour une question de ce type, que l'enquête porte sur plusieurs centaines d'élèves. Dans ce cas, et au prix d'une intervention interprétative guidée par les informations fournies par le logiciel, le pourcentage d'u.c.e. dans chaque classe d'analyse retenue après interprétation, est stable et semble bien être caractéristique du contenu informatif du corpus analysé.

Une autre précaution semble devoir être prise lorsqu'on utilise les u.c.e. les plus significatives de chaque classe pour l'étude d'un corpus hétérogène: elles ne sont pas toujours issues des élèves qui appartiennent au groupe le plus corrélé à la classe considérée.

\section{En guise de conclusion}

Malgré les limites inhérentes à la méthodologie utilisée (questionnaire suivi d'un traitement par un logiciel d'analyse de contenu), il semble bien que l'on puisse affirmer que les élèves de F2 se distinguent des autres élèves interrogés à l'occasion de cette recherche: partant d'une base commune en $2^{\text {ème }}$ (constatations immédiates et modélisation d'un dispositif électrique en termes de circuit), les approches se différencient en fonction de la section et du niveau scolaire.

61 Deux points importants peuvent être notés.

62 - D'une part, bien sûr, répondre à des questions « hors programme de physique $»^{28}$ pose problème aux élèves qui, en fonction des informations dont ils disposent sur le sujet, adoptent l'une ou l'autre des deux stratégies suivantes:

63 S'ils possèdent quelques informations (cas de la question $n^{\circ} 3$ pour les élèves de F2), ils peuvent tenter de répondre à l'inconnu en utilisant ce qu'ils connaissent. Par exemple, il semble bien que certains élèves de F2 appliquent à l'eau le modèle du «conducteur parfait ».

64 S'ils ne possèdent pas d'informations (cas de la question $n^{\circ} 4$ pour les élèves de F2), les élèves restent au niveau des constatations immédiates.

65 - D'autre part, il semble que l'on puisse envisager un effet secondaire résultant de la présence, dans une section, d'un programme de sciences physiques diversifié. Cet effet serait l'acquisition par les élèves d'une certaine aptitude à envisager un problème de manière différenciée. En effet, les élèves de F2 et ceux de SCE possèdent à peu près les mêmes informations relativement au problème proposé par la question $\mathrm{n}^{\circ} 2^{29}$; or, les réponses des élèves de SCE apparaissent comme plus complètes. Cela n'est pas sans poser la question des choix curriculaires qui permettraient aux élèves «du technique » de poursuivre des études scientifiques ou techniques au delà de « bac+2 ».

BIBLIOGRAPHIE 


\section{Références}

BOULDOIRES B., Les notations symboliques relatives à l'énergie dans quelques manuels de sciences physiques, in Actes du 1er Séminaire National de Recherche en Didactique des Sciences Physiques, U.J.F.-I.F.M., Grenoble, 1991.

LEBART L., SALEM A., Analyse statistique des données textuelles. Dunod-Bordas, Paris, 1988.

LEFEVRE R, ESCAUT A. MONCHOUX M-F. BIGNEBAT J., Sciences et techniques : Représentations des étudiants de 1ère Année Universitaire, in Pédagogiques, vol. 10, nº 2, 1992.

LEMEIGNAN G., WEIL-BARAIS A., Projet ENER, document interne LIRESPT, 1987 et Bilan du travail réalisé à propos d'énergie et projet, document interne LIRESPT. 1988.

REINERT M., Alceste une méthodologie d'analyse des données textuelles et une application : Aurélia de Gérard de Nerval, in Bulletin de Méthodologie Sociologique, A.I.M.S., n 26, mars 1990, pp. 24-54.

SPERBER D., WILSON D., La pertinence : Communication et cognition, Les Editions de Minuit. Paris. 1986. trad. fr. 1989.

\section{NOTES}

1. Cette recherche, financée par l'A.D.E.M.E. (Agence De l'Environnement et de la Maîtrise de l'Energie), complète une recherche antérieure relative aux notations symboliques utilisées dans les manuels scolaires pour représenter les grandeurs énergétiques (B. BOULDOIRES. 1991).

2. Pour les élèves de F2, section technique à vocation « électronique ", cette variable étant ellemême décomposable en deux « sous variables ", suivant que les élèves sont ou non titulaires d'un B.E.P.

3. Pour les élèves de 1ère $S$ et $E$, de Terminale $E$ et $C$, qui seront regroupés sous l'appellation commune SCE. On pourrait aussi ajouter la modalité « pas d'enseignement » (relatif à l'énergie au lycée) pour les élèves de seconde.

4. Logiciel développé par Max REINERT de l'université TOULOUSE-Le Mirail.

5. En référence aux travaux de D. SPERBER et D. WILSON (1986, trad. fr. 1989).

6. Cela n'est pas totalement neutre pour un traitement à l'aide d'ALCESTE car le découpage en unités de contexte élémentaires (u.c.e.) effectué par ce logiciel tient compte de la ponctuation (et de la longueur via le nombre de lignes).

7. Spontané en ce sens que rien ne les y incitait.

8. Au sens de D. SERBER et D. WILSON (op. cit.) : voir en particulier pages 182-183 (leur définition du concept). 227 et 230. ainsi que 233-242 (présentation du Principe de pertinence).

9. Bien que, au plan physique, on puisse envisager aussi bien une lampe qui ne s'allume pas qu'un bol d'eau qui se transforme en glace, la première situation est infiniment plus « raisonnable » que la seconde si on la relie aux expériences quotidiennes.

10. Forme réduite : la racine d'une famille de mots (exemple : compar+).

11. Il se peut en effet qu'un point de vue soit privilégié dans un sous-groupe d'élèves mais se retrouve également avec une fréquence importante dans d'autres sous-groupes. Dans ce cas, ce n'est pas le point de vue qui est caractéristique du sous-groupe mais le «renfermement» de certains élèves sur ce point de vue.

12. Analyse à l'aide d'un plan $\mathrm{SX} 12-16 / 12$ puis d'un plan $\mathrm{SX} 10-15 / 12$ et une longueur de ligne réduite à $80 \%$ du maximum. 
13. Le total des u.c.e. ne donne que $98 \%$ car une classe comportant cinq u.c.e. reste non interprétée.

14. Analyse à l'aide d'un plan SX16-14/10. Chaque fois que cela était possible, on a préféré donner les u.c.e. les plus caractéristiques de la classe plutôt qu'une interprétation. On a repéré en gras les formes les plus caractéristiques du profil lexical de la classe.

15. Associé à un raisonnement en termes de circulation de courant ou de charges, la transformation énergétique étant localisée au niveau de certains « éléments » du circuit sans que le transfert soit toujours explicité.

16. Associé à un transfert d'énergie. On pense là en particulier aux travaux de G. LEMEIGNAN et A. WEIL-BARAIS relatifs à l'enseignement de l'énergie dans les classes de 1ère.

17. Bien que, "a priori », ces élèves de $\mathrm{F} 2$ abordent également les problèmes énergétiques dans la partie «Mécanique » de l'enseignement «Etude des constructions » (voir B.O. Education Nationale, $\mathrm{N}^{\circ}$ spécial 1 du 6 avril 1989, p. 33 renvoyé p. 9; ainsi que le supplément au B.O. N 18, 12 mai 1988 Tome 1, p. 99).

18. Qui s'apparente à l'approche des élèves de Seconde.

19. Qui rappelle l'approche des élèves de F2.

20. Ou de conservation de l'énergie mécanique, approche que les élèves de SCE sont les seuls à entreprendre.

21. Bien qu'au cours d'une recherche antérieure (R. LEFEVRE. A. ESCAUT, M.F. MONCHOUX, J. BIGNEBAT, 1992), le thème « Energie » n'ait pas été abordé par les étudiants concernés.

22. Ce qui semble confirmé par les difficultés du post-codage d'où l'intérêt d'une telle approche qui ne masque pas cet aspect du problème comme pourrait le faire, par exemple, un questionnaire « fermé ».

23. a) «Le courant passe dans le circuit et l'énergie consommée fait briller la lampe » $\neq b$ ) «L'énergie passe dans le circuit et le courant consommé fait briller la lampe ». L'affirmation a) peut être considérée comme exacte, l'affirmation b) non. Pour les physiciens ces deux affirmations ne relèvent pas de la même conception. Il est par contre normal que le logiciel ALCESTE ne les distingue pas.

24. Soit que l'on interprète les résultats fournis par une analyse descendante hiérarchique pour choisir un découpage en classes "réaliste", soit que l'on découpe les réponses en u.c.e. constituant des « idées » au sens du physicien.

25. Le concepteur d'ALCESTE aborde d'ailleurs ces problèmes dans un article (REINERT M., 1990. voir en particulier pp. 29-32).

26. Choisi car c'est, parmi tous ceux que nous avons étudiés, celui qui présente la plus faible stabilité au plan des analyses.

27. Cette remarque limite donc la validité des analyses effectuées sur des sous-groupes dont les effectifs paraissent un peu faibles compte tenu du résultat précédent.

28. Calorimétrie et mécanique pour les élèves de la section F2.

29. Et c'est probablement au moins en partie à cause de cela qu'il est assez difficile d'utiliser ALCESTE pour étudier les réponses à cette question! 


\section{RÉSUMÉS}

Cette recherche concerne des élèves de lycée, de l'enseignement général scientifique et de l'enseignement technique français, âgés de 15 à 21 ans. Il s'agit de dégager, à propos de la notion d'Energie et en utilisant un logiciel d'analyse lexicale, l'influence de l'enseignement reçu. Une discussion méthodologique précise les limites de l'approche entreprise en différenciant les notions de "Conception" et "d'Univers de pensée".

This study concerns French pupils of scientific or technical secondary schools, aged 15 to 21 . It aims to point out, using lexical analysis software, the influence of Energy teaching contents. A methodological discussion emphasizes the limits of such an approach using a distinction between "Conception" and 'Mind Universe".

INDEX

Mots-clés : analyse lexicale, conception, énergie, enseignement général, enseignement technique, lycée, univers de pensée

\section{AUTEURS}

\section{B. BOULDOIRES}

Laboratoire d'étude des méthodes moderne d'enseignement, Université Paul Sabatier de Toulouse

\section{R. LEFEVRE}

Laboratoire d'étude des méthodes moderne d'enseignement, Université Paul Sabatier de Toulouse 